\title{
A New Impulsive Multi-Orders Fractional Differential Equation Involving Multipoint Fractional Integral Boundary Conditions
}

\author{
Guotao Wang, ${ }^{1}$ Sanyang Liu, ${ }^{1}$ Dumitru Baleanu, ${ }^{2,3,4}$ and Lihong $\mathrm{Zhang}^{5}$ \\ ${ }^{1}$ Department of Applied Mathematics, Xidian University, Xian, Shaanxi 710071, China \\ ${ }^{2}$ Department of Chemical and Materials Engineering, Faculty of Engineering, King Abdulaziz University, \\ P.O. Box 80204, Jeddah 21589, Saudi Arabia \\ ${ }^{3}$ Department of Mathematics and Computer Science, Çankaya University, 06530 Ankara, Turkey \\ ${ }^{4}$ Institute of Space Sciences, P.O. Box MG-23, 76900 Magurele-Bucharest, Romania \\ ${ }^{5}$ School of Mathematics and Computer Science, Shanxi Normal University, Linfen, Shanxi 041004, China \\ Correspondence should be addressed to Guotao Wang; wgt2512@163.com and Sanyang Liu; liusanyang@126.com
}

Received 6 April 2014; Accepted 24 May 2014; Published 16 June 2014

Academic Editor: J. C. Prajapati

Copyright (c) 2014 Guotao Wang et al. This is an open access article distributed under the Creative Commons Attribution License, which permits unrestricted use, distribution, and reproduction in any medium, provided the original work is properly cited.

A new impulsive multi-orders fractional differential equation is studied. The existence and uniqueness results are obtained for a nonlinear problem with fractional integral boundary conditions by applying standard fixed point theorems. An example for the illustration of the main result is presented.

\section{Introduction}

Nowadays, fractional differential equations have attracted a lot of attention due to its wide range of applications in many practical problems such as in physics, engineering, economics, and so on; see [1-5].

Impulsive differential equations have extensively been studied in the past two decades. Indeed impulsive differential equations are used to describe the dynamics of processes in which sudden, discontinuous jumps occur. Such processes are naturally seen in harvesting, earthquakes, diseases, and so forth. Recently, fractional impulsive differential equations have attracted the attention of many researchers. For the general theory and applications of such equations we refer the interested reader to see [6-24] and the references therein.

In this paper, we investigate a new impulsive nonlinear differential equation involving multi-orders fractional derivatives and deviating argument. Precisely, we consider the following multipoint fractional integral boundary value problem:

$$
\begin{array}{r}
{ }^{C} D_{t_{k}^{+}}^{\alpha_{k}} u(t)=f(t, u(t), u(\theta(t))), \quad 1<\alpha_{k} \leq 2, \\
k=0,1,2, \ldots, p, t \in J^{\prime},
\end{array}
$$

$$
\begin{array}{r}
\Delta u\left(t_{k}\right)=I_{k}\left(u\left(t_{k}\right)\right), \quad \Delta u^{\prime}\left(t_{k}\right)=I_{k}^{*}\left(u\left(t_{k}\right)\right), \\
k=1,2, \ldots, p, \\
u(0)=\sum_{k=0}^{p} \lambda_{k} \mathcal{F}_{t_{k}^{+}}^{\beta_{k}} u\left(\eta_{k}\right), \quad u^{\prime}(0)=0, \quad t_{k}<\eta_{k}<t_{k+1},
\end{array}
$$

where ${ }^{C} D_{t_{k}^{+}}^{\alpha_{k}}$ is the Caputo fractional derivative of order $\alpha_{k}$ and $\mathcal{F}_{t_{k}^{+}}^{\beta_{k}}$ is fractional Riemann-Liouville integral of order $\beta_{k}>0, f \in C(J \times \mathbb{R} \times \mathbb{R}, \mathbb{R}), I_{k}, I_{k}^{*} \in C(\mathbb{R}, \mathbb{R})$, deviating argument $\theta \in C(J, J), J=[0, T](T>0), 0=$ $t_{0}<t_{1}<\cdots<t_{k}<\cdots<t_{p}<t_{p+1}=T, J^{\prime}=$ $J /\left\{t_{1}, t_{2}, \ldots, t_{p}\right\}$, and $\Delta u\left(t_{k}\right)=u\left(t_{k}^{+}\right)-u\left(t_{k}^{-}\right)$, where $u\left(t_{k}^{+}\right)$and $u\left(t_{k}^{-}\right)$denote the right and the left limits of $u(t)$ at $t=t_{k}(k=$ $1,2, \ldots, p)$, respectively. $\Delta u^{\prime}\left(t_{k}\right)$ have a similar meaning for $u^{\prime}(t)$.

The paper is organized as follows. Section 2 gives some definitions and necessary lemmas, while the main results are presented in Section 3. 


\section{Preliminaries}

Let us fix $J_{0}=\left[0, t_{1}\right], J_{k-1}=\left(t_{k-1}, t_{k}\right]$, and $k=1,2, \ldots, p+1$ with $t_{p+1}=T$ and introduce a Banach space:

$$
P C(J, \mathbb{R})=\left\{u: J \longrightarrow \mathbb{R} \mid u \in C\left(J_{k}\right), k=0,1, \ldots, p,\right.
$$

$$
\left.u\left(t_{k}^{+}\right) \text {exist, } k=1,2, \ldots, p \cdot\right\},
$$

with the norm $\|u\|=\sup _{t \in J}|u(t)|$.

For the reader's convenience, we present some necessary definitions from fractional calculus theory and lemmas.

Definition 1. The Riemann-Liouville fractional integral of order $\alpha$ for a function $f:[d, \infty) \rightarrow \mathbb{R}$ is defined as

$$
\mathcal{F}_{d^{+}}^{\alpha} f(t)=\frac{1}{\Gamma(\alpha)} \int_{d}^{t}(t-s)^{\alpha-1} f(s) d s, \quad \alpha>0,
$$

provided the integral exists.

Definition 2. The Caputo fractional derivative of order $\alpha$ for a function $f:[d, \infty) \rightarrow \mathbb{R}$ is defined by

$$
{ }^{C} D_{d^{+}}^{\alpha} f(t)=\frac{1}{\Gamma(n-\alpha)} \int_{d}^{t}(t-s)^{n-\alpha-1} f^{(n)}(s) d s,
$$

where $[\alpha]$ denotes the integer part of real number $\alpha$.

Lemma 3. For a given $y \in C[0, T]$, a function $u$ is a solution of the following impulsive boundary value problem:

$$
\begin{gathered}
{ }^{C} D_{t_{k}^{+}}^{\alpha_{k}} u(t)=y(t), \quad 1<\alpha_{k} \leq 2, k=0,1,2, \ldots, p, t \in J^{\prime}, \\
\Delta u\left(t_{k}\right)=I_{k}\left(u\left(t_{k}\right)\right), \quad \Delta u^{\prime}\left(t_{k}\right)=I_{k}^{*}\left(u\left(t_{k}\right)\right), \\
k=1,2, \ldots, p, \\
u(0)=\sum_{k=0}^{p} \lambda_{k} \mathcal{F}_{t_{k}^{+}}^{\beta_{k}} u\left(\eta_{k}\right), \quad u^{\prime}(0)=0,
\end{gathered}
$$

if and only if $u$ is a solution of the impulsive fractional integral equation

$$
\left\{\begin{array}{l}
\frac{1}{\Gamma\left(\alpha_{0}\right)} \int_{0}^{t}(t-s)^{\alpha_{0}-1} y(s) d s+\mathscr{A}, \quad t \in J_{0} ; \\
\int_{t_{k}}^{t} \frac{(t-s)^{\alpha_{k}-1}}{\Gamma\left(\alpha_{k}\right)} y(s) d s \\
+\sum_{i=1}^{k}\left[\int_{t_{i-1}}^{t_{i}} \frac{\left(t_{i}-s\right)^{\alpha_{i-1}-1}}{\Gamma\left(\alpha_{i-1}\right)} y(s) d s+I_{i}\left(u\left(t_{i}\right)\right)\right] \\
+\sum_{i=1}^{k-1}\left(t_{k}-t_{i}\right)\left[\int_{t_{i-1}}^{t_{i}} \frac{\left(t_{i}-s\right)^{\alpha_{i-1}-2}}{\Gamma\left(\alpha_{i-1}-1\right)} y(s) d s\right. \\
\left.+I_{i}^{*}\left(u\left(t_{i}\right)\right)\right] \\
+\sum_{i=1}^{k}\left(t-t_{k}\right) \quad t \in J_{k}, k=1,2, \ldots, p, \\
\times\left[\int_{t_{i-1}}^{t_{i}} \frac{\left(t_{i}-s\right)^{\alpha_{i-1}-2}}{\Gamma\left(\alpha_{i-1}-1\right)} y(s) d s+I_{i}^{*}\left(u\left(t_{i}\right)\right)\right]+\mathscr{A},
\end{array}\right.
$$

where

$$
\begin{aligned}
& \mathscr{A}=(1\left.-\sum_{k=0}^{p} \frac{\lambda_{k}\left(\eta_{k}-t_{k}\right)^{\beta_{k}}}{\Gamma\left(\beta_{k}+1\right)}\right)^{-1} \\
& \times\left\{\sum_{k=0}^{p} \lambda_{k} \int_{t_{k}}^{\eta_{k}} \frac{\left(\eta_{k}-s\right)^{\alpha_{k}+\beta_{k}-1}}{\Gamma\left(\alpha_{k}+\beta_{k}\right)} y(s) d s\right. \\
&+\sum_{k=1 i=1}^{p} \sum_{i=1}^{k} \frac{\lambda_{k}\left(\eta_{k}-t_{k}\right)^{\beta_{k}}}{\Gamma\left(\beta_{k}+1\right)} \\
& \times\left[\int_{t_{i-1}}^{t_{i}} \frac{\left(t_{i}-s\right)^{\alpha_{i-1}-1}}{\Gamma\left(\alpha_{i-1}\right)} y(s) d s+I_{i}\left(u\left(t_{i}\right)\right)\right] \\
&+\sum_{k=1}^{p} \sum_{i=1}^{k-1} \frac{\lambda_{k}\left(\eta_{k}-t_{k}\right)^{\beta_{k}}\left(t_{k}-t_{i}\right)}{\Gamma\left(\beta_{k}+1\right)} \\
& \quad \times\left[\int_{t_{i-1}}^{t_{i}} \frac{\left(t_{i}-s\right)^{\alpha_{i-1}-2}}{\Gamma\left(\alpha_{i-1}-1\right)} y(s) d s+I_{i}^{*}\left(u\left(t_{i}\right)\right)\right] \\
&+\sum_{k=1}^{p} \sum_{i=1}^{k} \frac{\lambda_{k}\left(\eta_{k}-t_{k}\right)^{\beta_{k}+1}}{\Gamma\left(\beta_{k}+2\right)} \\
&\left.\times\left[\int_{t_{i-1}}^{t_{i}} \frac{\left(t_{i}-s\right)^{\alpha_{i-1}-2}}{\Gamma\left(\alpha_{i-1}-1\right)} y(s) d s+I_{i}^{*}\left(u\left(t_{i}\right)\right)\right]\right\} .
\end{aligned}
$$

Proof. Let $u$ be a solution of (5). For any $t \in J_{0}$, we have

$$
\begin{aligned}
u(t) & =\mathcal{J}_{0}^{\alpha_{0}} y(t)-c_{1}-c_{2} t \\
& =\frac{1}{\Gamma\left(\alpha_{0}\right)} \int_{0}^{t}(t-s)^{\alpha_{0}-1} y(s) d s-c_{1}-c_{2} t, \quad t \in J_{0},
\end{aligned}
$$


for some $c_{1}, c_{2} \in \mathbb{R}$. Differentiating (8), we get

$$
\begin{aligned}
& u^{\prime}(t)=\frac{1}{\Gamma\left(\alpha_{0}-1\right)} \int_{0}^{t}(t-s)^{\alpha_{0}-2} y(s) d s-c_{2}, \quad t \in J_{0} . \\
& \text { If } t \in J_{1} \text {, then } \\
& u(t)=\frac{1}{\Gamma\left(\alpha_{1}\right)} \int_{t_{1}}^{t}(t-s)^{\alpha_{1}-1} y(s) d s-d_{1}-d_{2}\left(t-t_{1}\right), \\
& u^{\prime}(t)=\frac{1}{\Gamma\left(\alpha_{1}-1\right)} \int_{t_{1}}^{t}(t-s)^{\alpha_{1}-2} y(s) d s-d_{2},
\end{aligned}
$$

for some $d_{1}, d_{2} \in \mathbb{R}$. Thus,

$$
\begin{aligned}
& u\left(t_{1}^{-}\right)=\frac{1}{\Gamma\left(\alpha_{0}\right)} \int_{0}^{t_{1}}\left(t_{1}-s\right)^{\alpha_{0}-1} y(s) d s-c_{1}-c_{2} t_{1}, \\
& u\left(t_{1}^{+}\right)=-d_{1}, \\
& u^{\prime}\left(t_{1}^{-}\right)=\frac{1}{\Gamma\left(\alpha_{0}-1\right)} \int_{0}^{t_{1}}\left(t_{1}-s\right)^{\alpha_{0}-2} y(s) d s-c_{2}, \\
& u^{\prime}\left(t_{1}^{+}\right)=-d_{2} .
\end{aligned}
$$

Using the impulse conditions

$$
\begin{gathered}
\Delta u\left(t_{1}\right)=u\left(t_{1}^{+}\right)-u\left(t_{1}^{-}\right)=I_{1}\left(u\left(t_{1}\right)\right), \\
\Delta u^{\prime}\left(t_{1}\right)=u^{\prime}\left(t_{1}^{+}\right)-u^{\prime}\left(t_{1}^{-}\right)=I_{1}^{*}\left(u\left(t_{1}\right)\right),
\end{gathered}
$$

we find that

$$
\begin{array}{r}
-d_{1} \\
=\frac{1}{\Gamma\left(\alpha_{0}\right)} \int_{0}^{t_{1}}\left(t_{1}-s\right)^{\alpha_{0}-1} y(s) d s-c_{1}-c_{2} t_{1}+I_{1}\left(u\left(t_{1}\right)\right), \\
-d_{2}=\frac{1}{\Gamma\left(\alpha_{0}-1\right)} \int_{0}^{t_{1}}\left(t_{1}-s\right)^{\alpha_{0}-2} y(s) d s-c_{2}+I_{1}^{*}\left(u\left(t_{1}\right)\right) .
\end{array}
$$

\section{Consequently,}

$$
\begin{aligned}
u(t)= & \frac{1}{\Gamma\left(\alpha_{1}\right)} \int_{t_{1}}^{t}(t-s)^{\alpha_{1}-1} y(s) d s \\
& +\frac{1}{\Gamma\left(\alpha_{0}\right)} \int_{0}^{t_{1}}\left(t_{1}-s\right)^{\alpha_{0}-1} y(s) d s \\
& +\frac{t-t_{1}}{\Gamma\left(\alpha_{0}-1\right)} \int_{0}^{t_{1}}\left(t_{1}-s\right)^{\alpha_{0}-2} y(s) d s \\
& +I_{1}\left(u\left(t_{1}\right)\right)+\left(t-t_{1}\right) I_{1}^{*}\left(u\left(t_{1}\right)\right)-c_{1}-c_{2} t, \\
& \quad t \in J_{1} .
\end{aligned}
$$

By a similar process, we can get

$$
\begin{aligned}
& u(t) \\
& =\int_{t_{k}}^{t} \frac{(t-s)^{\alpha_{k}-1}}{\Gamma\left(\alpha_{k}\right)} y(s) d s \\
& +\sum_{i=1}^{k}\left[\int_{t_{i-1}}^{t_{i}} \frac{\left(t_{i}-s\right)^{\alpha_{i-1}-1}}{\Gamma\left(\alpha_{i-1}\right)} y(s) d s+I_{i}\left(u\left(t_{i}\right)\right)\right] \\
& +\sum_{i=1}^{k-1}\left(t_{k}-t_{i}\right)\left[\int_{t_{i-1}}^{t_{i}} \frac{\left(t_{i}-s\right)^{\alpha_{i-1}-2}}{\Gamma\left(\alpha_{i-1}-1\right)} y(s) d s+I_{i}^{*}\left(u\left(t_{i}\right)\right)\right] \\
& \left.+\sum_{i=1}^{k}\left(t-t_{k}\right)\right] \\
& \quad \times\left[\int_{t_{i-1}}^{t_{i}} \frac{\left(t_{i}-s\right)^{\alpha_{i-1}-2}}{\Gamma\left(\alpha_{i-1}-1\right)} y(s) d s+I_{i}^{*}\left(u\left(t_{i}\right)\right)\right]-c_{1}-c_{2} t \\
&
\end{aligned}
$$

The boundary condition $u^{\prime}(0)=0$ implies $c_{2}=0$. For $t \in J_{k}$, we have

$$
\begin{aligned}
& \mathcal{J}_{t_{k}^{+}}^{\beta_{k}} u(t) \\
& =\int_{t_{k}}^{t} \frac{(t-s)^{\alpha_{k}+\beta_{k}-1}}{\Gamma\left(\alpha_{k}+\beta_{k}\right)} y(s) d s \\
& +\sum_{i=1}^{k} \frac{\left(t-t_{k}\right)^{\beta_{k}}}{\Gamma\left(\beta_{k}+1\right)}\left[\int_{t_{i-1}}^{t_{i}} \frac{\left(t_{i}-s\right)^{\alpha_{i-1}-1}}{\Gamma\left(\alpha_{i-1}\right)} y(s) d s+I_{i}\left(u\left(t_{i}\right)\right)\right] \\
& +\sum_{i=1}^{k-1} \frac{\left(t-t_{k}\right)^{\beta_{k}}\left(t_{k}-t_{i}\right)}{\Gamma\left(\beta_{k}+1\right)} \\
& \quad+\sum_{i=1}^{k} \frac{\left(t-t_{k}\right)^{\beta_{k}+1}}{\Gamma\left(\beta_{k}+2\right)}\left[\int_{t_{i-1}}^{t_{i}} \frac{\left(t_{i}-s\right)^{\alpha_{i-1}-2}}{\Gamma\left(\alpha_{i-1}-1\right)} y(s) d s+I_{i}^{*}\left(u\left(t_{i}\right)\right)\right. \\
& \quad-\frac{c_{1}\left(t-t_{k}\right)^{\beta_{k}}}{\Gamma\left(\beta_{k}+1\right)}, \\
& \quad+\sum_{k=0}^{p} \lambda_{k} \int_{t_{k}}^{\eta_{i-1}-2} \frac{\left(\eta_{k}-s\right)^{\alpha_{k}+\beta_{k}-1}}{\Gamma\left(\alpha_{k}+\beta_{k}\right)} y(s) d s \\
& \sum_{k=0}^{p} \lambda_{k} \mathcal{F}_{t_{k}^{+}}^{\beta_{k}} u\left(\eta_{k}\right) \\
& \left.\quad \sum_{k=1}^{p} \frac{\lambda_{k}\left(\eta_{k}-t_{k}\right)^{\beta_{k}}}{\Gamma\left(\beta_{k}+1\right)} I_{i}^{*}\left(u\left(t_{i}\right)\right)\right]
\end{aligned}
$$




$$
\begin{aligned}
& \times\left[\int_{t_{i-1}}^{t_{i}} \frac{\left(t_{i}-s\right)^{\alpha_{i-1}-1}}{\Gamma\left(\alpha_{i-1}\right)} y(s) d s+I_{i}\left(u\left(t_{i}\right)\right)\right] \\
& +\sum_{k=1}^{p} \sum_{i=1}^{k-1} \frac{\lambda_{k}\left(\eta_{k}-t_{k}\right)^{\beta_{k}}\left(t_{k}-t_{i}\right)}{\Gamma\left(\beta_{k}+1\right)} \\
& \times\left[\int_{t_{i-1}}^{t_{i}} \frac{\left(t_{i}-s\right)^{\alpha_{i-1}-2}}{\Gamma\left(\alpha_{i-1}-1\right)} y(s) d s+I_{i}^{*}\left(u\left(t_{i}\right)\right)\right] \\
& +\sum_{k=1}^{p} \sum_{i=1}^{k} \frac{\lambda_{k}\left(\eta_{k}-t_{k}\right)^{\beta_{k}+1}}{\Gamma\left(\beta_{k}+2\right)} \\
& \quad \times\left[\int_{t_{i-1}}^{t_{i}} \frac{\left(t_{i}-s\right)^{\alpha_{i-1}-2}}{\Gamma\left(\alpha_{i-1}-1\right)} y(s) d s+I_{i}^{*}\left(u\left(t_{i}\right)\right)\right] \\
& -\sum_{k=0}^{p} \frac{c_{1} \lambda_{k}\left(\eta_{k}-t_{k}\right)^{\beta_{k}}}{\Gamma\left(\beta_{k}+1\right)} .
\end{aligned}
$$

Applying the boundary condition $u(0)=\sum_{k=0}^{p} \lambda_{k}$ $\mathcal{F}_{t_{k}^{+}}^{\beta_{k}} u\left(\eta_{k}\right)$, then

$$
\begin{aligned}
-c_{1}=(1- & \left.\sum_{k=0}^{p} \frac{\lambda_{k}\left(\eta_{k}-t_{k}\right)^{\beta_{k}}}{\Gamma\left(\beta_{k}+1\right)}\right)^{-1} \\
& \times\left\{\sum_{k=0}^{p} \lambda_{k} \int_{t_{k}}^{\eta_{k}} \frac{\left(\eta_{k}-s\right)^{\alpha_{k}+\beta_{k}-1}}{\Gamma\left(\alpha_{k}+\beta_{k}\right)} y(s) d s\right. \\
& +\sum_{k=1}^{p} \sum_{i=1}^{k} \frac{\lambda_{k}\left(\eta_{k}-t_{k}\right)^{\beta_{k}}}{\Gamma\left(\beta_{k}+1\right)} \\
& \times\left[\int_{t_{i-1}}^{t_{i}} \frac{\left(t_{i}-s\right)^{\alpha_{i-1}-1}}{\Gamma\left(\alpha_{i-1}\right)} y(s) d s+I_{i}\left(u\left(t_{i}\right)\right)\right] \\
& +\sum_{k=1}^{p} \sum_{i=1}^{k-1} \frac{\lambda_{k}\left(\eta_{k}-t_{k}\right)^{\beta_{k}}\left(t_{k}-t_{i}\right)}{\Gamma\left(\beta_{k}+1\right)} \\
& \times\left[\int_{t_{i-1}}^{t_{i}} \frac{\left(t_{i}-s\right)^{\alpha_{i-1}-2}}{\Gamma\left(\alpha_{i-1}-1\right)} y(s) d s+I_{i}^{*}\left(u\left(t_{i}\right)\right)\right] \\
& +\sum_{k=1}^{p} \sum_{i=1}^{k} \frac{\lambda_{k}\left(\eta_{k}-t_{k}\right)^{\beta_{k}+1}}{\Gamma\left(\beta_{k}+2\right)} \\
& \left.\times\left[\int_{t_{i-1}}^{t_{i}} \frac{\left(t_{i}-s\right)^{\alpha_{i-1}-2}}{\Gamma\left(\alpha_{i-1}-1\right)} y(s) d s+I_{i}^{*}\left(u\left(t_{i}\right)\right)\right]\right\} .
\end{aligned}
$$

Substituting the value of $c_{i}(i=1,2)$ in (8) and (15), we obtain (6). Conversely, assume that $u$ is a solution of the impulsive fractional integral equation (6); then by a direct computation, it follows that the solution given by (6) satisfies (5). This completes the proof.

\section{Main Results}

Define an operator $\mathscr{G}: P C(J, \mathbb{R}) \rightarrow P C(J, \mathbb{R})$ by

$\mathscr{G} u(t)$

$$
\begin{aligned}
= & \int_{t_{k}}^{t} \frac{(t-s)^{\alpha_{k}-1}}{\Gamma\left(\alpha_{k}\right)} f(s, u(s), u(\theta(s))) d s \\
& +\sum_{i=1}^{k} \\
& \times\left[\int_{t_{i-1}}^{t_{i}} \frac{\left(t_{i}-s\right)^{\alpha_{i-1}-1}}{\Gamma\left(\alpha_{i-1}\right)} f(s, u(s), u(\theta(s))) d s+I_{i}\left(u\left(t_{i}\right)\right)\right] \\
& +\sum_{i=1}^{k-1}\left(t_{k}-t_{i}\right) \\
& \times\left[\int_{t_{i-1}}^{t_{i}} \frac{\left(t_{i}-s\right)^{\alpha_{i-1}-2}}{\Gamma\left(\alpha_{i-1}-1\right)} f(s, u(s), u(\theta(s))) d s+I_{i}^{*}\left(u\left(t_{i}\right)\right)\right]
\end{aligned}
$$$$
+\sum_{i=1}^{k}\left(t-t_{k}\right)
$$$$
\times\left[\int_{t_{i-1}}^{t_{i}} \frac{\left(t_{i}-s\right)^{\alpha_{i-1}-2}}{\Gamma\left(\alpha_{i-1}-1\right)} f(s, u(s), u(\theta(s))) d s+I_{i}^{*}\left(u\left(t_{i}\right)\right)\right]
$$$$
+\left(1-\sum_{k=0}^{p} \frac{\lambda_{k}\left(\eta_{k}-t_{k}\right)^{\beta_{k}}}{\Gamma\left(\beta_{k}+1\right)}\right)^{-1}
$$$$
\times\left\{\sum_{k=0}^{p} \lambda_{k} \int_{t_{k}}^{\eta_{k}} \frac{\left(\eta_{k}-s\right)^{\alpha_{k}+\beta_{k}-1}}{\Gamma\left(\alpha_{k}+\beta_{k}\right)} f(s, u(s), u(\theta(s))) d s\right.
$$

$$
\begin{aligned}
& +\sum_{k=1}^{p} \sum_{i=1}^{k} \frac{\lambda_{k}\left(\eta_{k}-t_{k}\right)^{\beta_{k}}}{\Gamma\left(\beta_{k}+1\right)} \\
& \times\left[\int_{t_{i-1}}^{t_{i}} \frac{\left(t_{i}-s\right)^{\alpha_{i-1}-1}}{\Gamma\left(\alpha_{i-1}\right)} f(s, u(s), u(\theta(s))) d s\right. \\
& \left.\quad+I_{i}\left(u\left(t_{i}\right)\right)\right] \\
& +\sum_{k=1}^{p} \sum_{i=1}^{k-1} \frac{\lambda_{k}\left(\eta_{k}-t_{k}\right)^{\beta_{k}}\left(t_{k}-t_{i}\right)}{\Gamma\left(\beta_{k}+1\right)} \\
& \times\left[\int_{t_{i-1}}^{t_{i}} \frac{\left(t_{i}-s\right)^{\alpha_{i-1}-2}}{\Gamma\left(\alpha_{i-1}-1\right)} f(s, u(s), u(\theta(s))) d s\right.
\end{aligned}
$$

$$
\begin{gathered}
\left.+I_{i}^{*}\left(u\left(t_{i}\right)\right)\right] \\
+\sum_{k=1}^{p} \sum_{i=1}^{k} \frac{\lambda_{k}\left(\eta_{k}-t_{k}\right)^{\beta_{k}+1}}{\Gamma\left(\beta_{k}+2\right)}
\end{gathered}
$$




$$
\begin{gathered}
\times\left[\int_{t_{i-1}}^{t_{i}} \frac{\left(t_{i}-s\right)^{\alpha_{i-1}-2}}{\Gamma\left(\alpha_{i-1}-1\right)} f(s, u(s), u(\theta(s))) d s\right. \\
\left.\left.\quad+I_{i}^{*}\left(u\left(t_{i}\right)\right)\right]\right\} .
\end{gathered}
$$

Notice that problem (1) has a solution if and only if the operator $\mathscr{G}$ has a fixed point.

For convenience, we will give some notations:

$$
\begin{aligned}
T^{*} & =\max _{0 \leq i \leq p}\left\{T^{\alpha_{i}}\right\}, \quad \Gamma^{*}=\min _{0 \leq i \leq p}\left\{\Gamma\left(\alpha_{i}\right)\right\}, \\
\Lambda_{1} & =\sum_{k=0}^{p} \frac{\lambda_{k} T^{\alpha_{k}+\beta_{k}}}{\Gamma\left(\alpha_{k}+\beta_{k}+1\right)}, \quad \Lambda_{2}=\sum_{k=1}^{p} \frac{\lambda_{k} T^{\beta_{k}}}{\Gamma\left(\beta_{k}+1\right)}, \\
\Lambda_{3} & =\sum_{k=1}^{p} \frac{\lambda_{k} T^{\beta_{k}}}{\Gamma\left(\beta_{k}+2\right)}, \quad \Delta=\left|1-\sum_{k=0}^{p} \frac{\lambda_{k}\left(\eta_{k}-t_{k}\right)^{\beta_{k}}}{\Gamma\left(\beta_{k}+1\right)}\right|^{-1}, \\
\Upsilon & =\frac{3 p T^{*}}{\Gamma^{*}}+\Delta\left[\Lambda_{1}+\frac{(2 p-1) T^{*}}{\Gamma^{*}} \Lambda_{2}+\frac{p T^{*}}{\Gamma^{*}} \Lambda_{3}\right], \\
\mu(x) & =\Upsilon\|x\|+\left(1+\Lambda_{2}\right) p L_{2}+\left[(p-1) T \Lambda_{2}+p T \Lambda_{3}\right] L_{3} .
\end{aligned}
$$

Theorem 4. Assume the following.

(H1) There exists a nonnegative function $a(t) \in L(0, T)$ such that

$$
|f(t, u, v)| \leq a(t)+\xi_{1}|u|^{\rho}+\xi_{2}|v|^{\varrho}, \quad 0<\rho, \varrho<1,
$$

where $\xi_{1}, \xi_{2}$ are nonnegative constants.

(H2) There exist positive constants $L_{2}$ and $L_{3}$ such that

$\left|I_{k}(u)\right| \leq L_{2}, \quad\left|I_{k}^{*}(u)\right| \leq L_{3}, \quad$ for $t \in J, u \in \mathbb{R}$,

$$
k=1,2, \ldots, p \text {. }
$$

Then problem (1) has at least one solution.

Proof. Firstly, we will prove that $\mathscr{G}: P C(J, \mathbb{R}) \rightarrow P C(J, \mathbb{R})$ is a completely continuous operator. Obviously, the continuity of functions $f, I_{k}$, and $I_{k}^{*}$ ensures the continuity of operator $\mathscr{G}$.

Let $\Omega \subset P C(J, \mathbb{R})$ be bounded. Then, there exist positive constants $L_{i}>0(i=1,2,3)$ such that $|f(t, u)| \leq L_{1}$,
$\left|I_{k}(u)\right| \leq L_{2}$ and $\left|I_{k}^{*}(u)\right| \leq L_{3}$ for all $u \in \Omega$. Thus, for any $u \in \Omega$, we have

$|\mathscr{G} u(t)|$

$\leq \int_{t_{k}}^{t} \frac{(t-s)^{\alpha_{k}-1}}{\Gamma\left(\alpha_{k}\right)}|f(s, u(s), u(\theta(s)))| d s$

$+\sum_{i=1}^{k}\left[\int_{t_{i-1}}^{t_{i}} \frac{\left(t_{i}-s\right)^{\alpha_{i-1}-1}}{\Gamma\left(\alpha_{i-1}\right)}|f(s, u(s), u(\theta(s)))| d s\right.$

$$
\left.+\left|I_{i}\left(u\left(t_{i}\right)\right)\right|\right]
$$

$+\sum_{i=1}^{k-1}\left(t_{k}-t_{i}\right)$

$\times\left[\int_{t_{i-1}}^{t_{i}} \frac{\left(t_{i}-s\right)^{\alpha_{i-1}-2}}{\Gamma\left(\alpha_{i-1}-1\right)}|f(s, u(s), u(\theta(s)))| d s\right.$

$$
\left.+\left|I_{i}^{*}\left(u\left(t_{i}\right)\right)\right|\right]
$$

$+\sum_{i=1}^{k}\left(t-t_{k}\right)$

$\times\left[\int_{t_{i-1}}^{t_{i}} \frac{\left(t_{i}-s\right)^{\alpha_{i-1}-2}}{\Gamma\left(\alpha_{i-1}-1\right)}|f(s, u(s), u(\theta(s)))| d s\right.$

$$
\begin{gathered}
\left.+\left|I_{i}^{*}\left(u\left(t_{i}\right)\right)\right|\right] \\
+\left|1-\sum_{k=0}^{p} \frac{\lambda_{k}\left(\eta_{k}-t_{k}\right)^{\beta_{k}}}{\Gamma\left(\beta_{k}+1\right)}\right|^{-1} \\
\times\left\{\sum_{k=0}^{p} \lambda_{k} \int_{t_{k}}^{\eta_{k}} \frac{\left(\eta_{k}-s\right)^{\alpha_{k}+\beta_{k}-1}}{\Gamma\left(\alpha_{k}+\beta_{k}\right)}|f(s, u(s), u(\theta(s)))| d s\right. \\
+\sum_{k=1}^{p} \sum_{i=1}^{k} \frac{\lambda_{k}\left(\eta_{k}-t_{k}\right)^{\beta_{k}}}{\Gamma\left(\beta_{k}+1\right)} \\
\times\left[\int_{t_{i-1}}^{t_{i}} \frac{\left(t_{i}-s\right)^{\alpha_{i-1}-1}}{\Gamma\left(\alpha_{i-1}\right)}|f(s, u(s), u(\theta(s)))| d s\right. \\
+\sum_{k=1}^{p} \frac{\sum_{i=1}^{k-1} \frac{\lambda_{k}\left(\eta_{k}-t_{k}\right)^{\beta_{k}}\left(t_{k}-t_{i}\right)}{\Gamma\left(\beta_{k}+1\right)}}{\times\left[\int_{t_{i-1}}^{t_{i}} \frac{\left(t_{i}-s\right)^{\alpha_{i-1}-2}}{\Gamma\left(\alpha_{i-1}-1\right)}|f(s, u(s), u(\theta(s)))| d s\right.} \\
+\left|I_{i}^{*}\left(u\left(t_{i}\right)\right)\right|
\end{gathered}
$$




$$
\begin{aligned}
& +\sum_{k=1}^{p} \sum_{i=1}^{k} \frac{\lambda_{k}\left(\eta_{k}-t_{k}\right)^{\beta_{k}+1}}{\Gamma\left(\beta_{k}+2\right)} \\
& \times\left[\int_{t_{i-1}}^{t_{i}} \frac{\left(t_{i}-s\right)^{\alpha_{i-1}-2}}{\Gamma\left(\alpha_{i-1}-1\right)}|f(s, u(s), u(\theta(s)))| d s\right. \\
& \left.\left.+\left|I_{i}^{*}\left(u\left(t_{i}\right)\right)\right|\right]\right\} \\
& \leq L_{1} \frac{\left(t-t_{k}\right)^{\alpha_{k}}}{\Gamma\left(\alpha_{k}+1\right)}+\sum_{i=1}^{k}\left[L_{1} \frac{\left(t_{i}-t_{i-1}\right)^{\alpha_{i-1}}}{\Gamma\left(\alpha_{i-1}+1\right)}+L_{2}\right] \\
& +\sum_{i=1}^{k-1}\left(t_{k}-t_{i}\right)\left[L_{1} \frac{\left(t_{i}-t_{i-1}\right)^{\alpha_{i-1}-1}}{\Gamma\left(\alpha_{i-1}\right)}+L_{3}\right] \\
& +\sum_{i=1}^{k}\left(t-t_{k}\right)\left[L_{1} \frac{\left(t_{i}-t_{i-1}\right)^{\alpha_{i-1}-1}}{\Gamma\left(\alpha_{i-1}\right)}+L_{3}\right] \\
& +\Delta\left\{L_{1} \sum_{k=0}^{p} \frac{\lambda_{k}\left(\eta_{k}-t_{k}\right)^{\alpha_{k}+\beta_{k}}}{\Gamma\left(\alpha_{k}+\beta_{k}+1\right)}\right. \\
& +\sum_{k=1}^{p} \sum_{i=1}^{k} \frac{\lambda_{k}\left(\eta_{k}-t_{k}\right)^{\beta_{k}}}{\Gamma\left(\beta_{k}+1\right)}\left[L_{1} \frac{\left(t_{i}-t_{i-1}\right)^{\alpha_{i-1}}}{\Gamma\left(\alpha_{i-1}+1\right)}+L_{2}\right] \\
& +\sum_{k=1}^{p} \sum_{i=1}^{k-1} \frac{\lambda_{k}\left(\eta_{k}-t_{k}\right)^{\beta_{k}}\left(t_{k}-t_{i}\right)}{\Gamma\left(\beta_{k}+1\right)} \\
& \times\left[L_{1} \frac{\left(t_{i}-t_{i-1}\right)^{\alpha_{i-1}-1}}{\Gamma\left(\alpha_{i-1}\right)}+L_{3}\right] \\
& \left.+\sum_{k=1}^{p} \sum_{i=1}^{k} \frac{\lambda_{k}\left(\eta_{k}-t_{k}\right)^{\beta_{k}+1}}{\Gamma\left(\beta_{k}+2\right)}\left[L_{1} \frac{\left(t_{i}-t_{i-1}\right)^{\alpha_{i-1}-1}}{\Gamma\left(\alpha_{i-1}\right)}+L_{3}\right]\right\} \\
& \leq L_{1} \sum_{i=1}^{p+1} \frac{\left(t_{i}-t_{i-1}\right)^{\alpha_{i-1}}}{\Gamma\left(\alpha_{i-1}+1\right)}+p L_{2} \\
& +T L_{1} \sum_{i=1}^{p-1} \frac{\left(t_{i}-t_{i-1}\right)^{\alpha_{i-1}-1}}{\Gamma\left(\alpha_{i-1}\right)}+(p-1) T L_{3} \\
& +T L_{1} \sum_{i=1}^{p} \frac{\left(t_{i}-t_{i-1}\right)^{\alpha_{i-1}-1}}{\Gamma\left(\alpha_{i-1}\right)}+p T L_{3} \\
& +\Delta\left\{L_{1} \sum_{k=0}^{p} \frac{\lambda_{k}\left(\eta_{k}-t_{k}\right)^{\alpha_{k}+\beta_{k}}}{\Gamma\left(\alpha_{k}+\beta_{k}+1\right)}\right. \\
& +L_{1} \sum_{k=1}^{p} \sum_{i=1}^{k} \frac{\lambda_{k}\left(\eta_{k}-t_{k}\right)^{\beta_{k}}\left(t_{i}-t_{i-1}\right)^{\alpha_{i-1}}}{\Gamma\left(\beta_{k}+1\right) \Gamma\left(\alpha_{i-1}+1\right)} \\
& +L_{2} \sum_{k=1}^{p} \sum_{i=1}^{k} \frac{\lambda_{k}\left(\eta_{k}-t_{k}\right)^{\beta_{k}}}{\Gamma\left(\beta_{k}+1\right)} \\
& +L_{1} \sum_{k=1}^{p} \sum_{i=1}^{k-1} \frac{\lambda_{k}\left(\eta_{k}-t_{k}\right)^{\beta_{k}}\left(t_{k}-t_{i}\right)\left(t_{i}-t_{i-1}\right)^{\alpha_{i-1}-1}}{\Gamma\left(\beta_{k}+1\right) \Gamma\left(\alpha_{i-1}\right)}
\end{aligned}
$$

$$
\begin{aligned}
& +L_{3} \sum_{k=1}^{p} \sum_{i=1}^{k-1} \frac{\lambda_{k}\left(\eta_{k}-t_{k}\right)^{\beta_{k}}\left(t_{k}-t_{i}\right)}{\Gamma\left(\beta_{k}+1\right)} \\
& +L_{1} \sum_{k=1}^{p} \sum_{i=1}^{k} \frac{\lambda_{k}\left(\eta_{k}-t_{k}\right)^{\beta_{k}+1}\left(t_{i}-t_{i-1}\right)^{\alpha_{i-1}-1}}{\Gamma\left(\beta_{k}+2\right) \Gamma\left(\alpha_{i-1}\right)} \\
& \left.+L_{3} \sum_{k=1}^{p} \sum_{i=1}^{k} \frac{\lambda_{k}\left(\eta_{k}-t_{k}\right)^{\beta_{k}+1}}{\Gamma\left(\beta_{k}+2\right)}\right\} \\
& \leq L_{1} \sum_{i=1}^{p+1} \frac{T^{*}}{\Gamma^{*}}+p L_{2}+T L_{1} \sum_{i=1}^{p-1} \frac{T^{*}}{\Gamma^{*}} \\
& +(p-1) T L_{3}+T L_{1} \sum_{i=1}^{p} \frac{T^{*}}{\Gamma^{*}}+p T L_{3} \\
& +\Delta\left\{L_{1} \sum_{k=0}^{p} \frac{\lambda_{k} T^{\alpha_{k}+\beta_{k}}}{\Gamma\left(\alpha_{k}+\beta_{k}+1\right)}+p L_{1}\right. \\
& \times \sum_{k=1}^{p} \frac{\lambda_{k} T^{\beta_{k}} T^{*}}{\Gamma\left(\beta_{k}+1\right) \Gamma^{*}}+p L_{2} \sum_{k=1}^{p} \frac{\lambda_{k} T^{\beta_{k}}}{\Gamma\left(\beta_{k}+1\right)} \\
& +(p-1) L_{1} \sum_{k=1}^{p} \frac{\lambda_{k} T^{\beta_{k}} T^{*}}{\Gamma\left(\beta_{k}+1\right) \Gamma^{*}} \\
& +(p-1) L_{3} \sum_{k=1}^{p} \frac{\lambda_{k} T^{\beta_{k}+1}}{\Gamma\left(\beta_{k}+1\right)} \\
& \left.+p L_{1} \sum_{k=1}^{p} \frac{\lambda_{k} T^{\beta_{k}} T^{*}}{\Gamma\left(\beta_{k}+2\right) \Gamma^{*}}+p L_{3} \sum_{k=1}^{p} \frac{\lambda_{k} T^{\beta_{k}+1}}{\Gamma\left(\beta_{k}+2\right)}\right\} \\
& \leq\left\{\frac{3 p T^{*}}{\Gamma^{*}}+\Delta\left[\Lambda_{1}+\frac{(2 p-1) T^{*}}{\Gamma^{*}} \Lambda_{2}+\frac{p T^{*}}{\Gamma^{*}} \Lambda_{3}\right]\right\} L_{1} \\
& +\left(1+\Lambda_{2}\right) p L_{2}+\left[(p-1) T \Lambda_{2}+p T \Lambda_{3}\right] L_{3} \\
& :=\Upsilon L_{1}+\left(1+\Lambda_{2}\right) p L_{2}+\left[(p-1) T \Lambda_{2}+p T \Lambda_{3}\right] L_{3} \text {, }
\end{aligned}
$$

which implies

$$
\begin{aligned}
\|\mathscr{G} u\| & \leq \Upsilon L_{1}+\left(1+\Lambda_{2}\right) p L_{2}+\left[(p-1) T \Lambda_{2}+p T \Lambda_{3}\right] L_{3} \\
& :=\mathscr{L} \text { (constant) }
\end{aligned}
$$

On the other hand, for any $t \in J_{k}, 0 \leq k \leq p$, we have

$\left|(\mathscr{G} u)^{\prime}(t)\right|$

$$
\begin{aligned}
\leq & \int_{t_{k}}^{t} \frac{(t-s)^{\alpha_{k}-2}}{\Gamma\left(\alpha_{k}-1\right)}|f(s, u(s), u(\theta(s)))| d s \\
& +\sum_{i=1}^{k}\left[\int_{t_{i-1}}^{t_{i}} \frac{\left(t_{i}-s\right)^{\alpha_{i-1}-2}}{\Gamma\left(\alpha_{i-1}-1\right)}|f(s, u(s), u(\theta(s)))| d s\right.
\end{aligned}
$$

$$
\left.+\left|I_{i}^{*}\left(u\left(t_{i}\right)\right)\right|\right]
$$




$$
\begin{aligned}
& \leq L_{1} \int_{t_{k}}^{t} \frac{(t-s)^{\alpha_{k}-2}}{\Gamma\left(\alpha_{k}-1\right)} d s+\sum_{i=1}^{p}\left[L_{1} \int_{t_{i-1}}^{t_{i}} \frac{\left(t_{i}-s\right)^{\alpha_{i-1}-2}}{\Gamma\left(\alpha_{i-1}-1\right)} d s+L_{3}\right] \\
& \leq \frac{T^{\alpha_{k}-1} L_{1}}{\Gamma\left(\alpha_{k}\right)}+p\left[\frac{L_{1} \max _{0 \leq i \leq p} T^{\alpha_{i}-1}}{\min _{0 \leq i \leq p} \Gamma\left(\alpha_{i}\right)}+L_{3}\right] \\
& \leq(p+1) \frac{L_{1} T^{*}}{T \Gamma^{*}}+p L_{3}:=\mathfrak{Q} \text { (constant). }
\end{aligned}
$$

Hence, for $\tau_{1}, \tau_{2} \in J_{k}$ with $\tau_{1} \leq \tau_{2}$ and $0 \leq k \leq p$, we have

$$
\left|(\mathscr{G} u)\left(\tau_{2}\right)-(\mathscr{G} u)\left(\tau_{1}\right)\right| \leq \int_{\tau_{1}}^{\tau_{2}}\left|(\mathscr{G} u)^{\prime}(s)\right| d s \leq \mathfrak{Q}\left(t_{2}-t_{1}\right) .
$$

This implies that $\mathscr{G} u$ is equicontinuous on all $J_{k}, k=$ $0,1,2, \ldots, p$. Consequently, Arzela-Ascoli theorem ensures the operator $\mathscr{G}: P C(J, \mathbb{R}) \rightarrow P C(J, \mathbb{R})$ is a completely continuous operator.

Next, we will show that the operator $\mathscr{G}$ maps $\mathscr{B}$ into $\mathscr{B}$. For that, let us choose $R \geq \max \left\{3 \mu,\left(3 \curlyvee \xi_{1}\right)^{(1 /(1-\rho))}\right.$, $\left.\left(3 \Upsilon \xi_{2}\right)^{(1 /(1-\varrho))}\right\}$ and define a ball $\mathscr{B}=\{u \in P C(J, \mathbb{R}):\|u\| \leq$ $R\}$. For any $u \in \mathscr{B}$, by the conditions $\left(\mathbf{H}_{\mathbf{1}}\right)$ and $\left(\mathbf{H}_{2}\right)$, we have

$|\mathscr{G} u(t)|$

$$
\begin{gathered}
\leq \int_{t_{k}}^{t} \frac{(t-s)^{\alpha_{k}-1}}{\Gamma\left(\alpha_{k}\right)}\left[a(s)+\xi_{1}|u(s)|^{\rho}+\xi_{2}|u(\theta(s))|^{\rho}\right] d s \\
+\sum_{i=1}^{k}\left[\int_{t_{i-1}}^{t_{i}} \frac{\left(t_{i}-s\right)^{\alpha_{i-1}-1}}{\Gamma\left(\alpha_{i-1}\right)}\right. \\
\times\left[a(s)+\xi_{1}|u(s)|^{\rho}+\xi_{2}|u(\theta(s))|^{\varrho}\right] d s \\
\left.+\left|I_{i}\left(u\left(t_{i}\right)\right)\right|\right] \\
+\sum_{i=1}^{k-1}\left(t_{k}-t_{i}\right)\left[\int_{t_{i-1}}^{t_{i}} \frac{\left(t_{i}-s\right)^{\alpha_{i-1}-2}}{\Gamma\left(\alpha_{i-1}-1\right)}\right. \\
\times\left[a(s)+\xi_{1}|u(s)|^{\rho}+\xi_{2}|u(\theta(s))|^{\varrho}\right] d s \\
\left.+\left|I_{i}^{*}\left(u\left(t_{i}\right)\right)\right|\right] \\
+\sum_{i=1}^{k}\left(t-t_{k}\right)\left[\int_{t_{i-1}}^{t_{i}} \frac{\left(t_{i}-s\right)^{\alpha_{i-1}-2}}{\Gamma\left(\alpha_{i-1}-1\right)}\right. \\
\times\left[a(s)+\xi_{1}|u(s)|^{\rho}+\xi_{2}|u(\theta(s))|^{\varrho}\right] d s \\
\left.+\left|I_{i}^{*}\left(u\left(t_{i}\right)\right)\right|\right]
\end{gathered}
$$

$$
\begin{aligned}
& +\left|1-\sum_{k=0}^{p} \frac{\lambda_{k}\left(\eta_{k}-t_{k}\right)^{\beta_{k}}}{\Gamma\left(\beta_{k}+1\right)}\right|^{-1} \\
& \times\left\{\sum_{k=0}^{p} \lambda_{k} \int_{t_{k}}^{\eta_{k}} \frac{\left(\eta_{k}-s\right)^{\alpha_{k}+\beta_{k}-1}}{\Gamma\left(\alpha_{k}+\beta_{k}\right)}\right. \\
& \quad \times\left[a(s)+\xi_{1}|u(s)|^{\rho}+\xi_{2}|u(\theta(s))|^{\rho}\right] d s \\
& +\sum_{k=1}^{p} \sum_{i=1}^{k} \frac{\lambda_{k}\left(\eta_{k}-t_{k}\right)^{\beta_{k}}}{\Gamma\left(\beta_{k}+1\right)}
\end{aligned}
$$$$
\times\left[\int_{t_{i-1}}^{t_{i}} \frac{\left(t_{i}-s\right)^{\alpha_{i-1}-1}}{\Gamma\left(\alpha_{i-1}\right)}\right.
$$$$
\times\left[a(s)+\xi_{1}|u(s)|^{\rho}+\xi_{2}|u(\theta(s))|^{\varrho}\right] d s
$$$$
\left.+\left|I_{i}\left(u\left(t_{i}\right)\right)\right|\right]
$$$$
+\sum_{k=1}^{p} \sum_{i=1}^{k-1} \frac{\lambda_{k}\left(\eta_{k}-t_{k}\right)^{\beta_{k}}\left(t_{k}-t_{i}\right)}{\Gamma\left(\beta_{k}+1\right)}
$$$$
\times\left[\int_{t_{i-1}}^{t_{i}} \frac{\left(t_{i}-s\right)^{\alpha_{i-1}-2}}{\Gamma\left(\alpha_{i-1}-1\right)}\right.
$$$$
\times\left[a(s)+\xi_{1}|u(s)|^{\rho}+\xi_{2}|u(\theta(s))|^{\rho}\right] d s
$$$$
\left.+\left|I_{i}^{*}\left(u\left(t_{i}\right)\right)\right|\right]
$$$$
+\sum_{k=1}^{p} \sum_{i=1}^{k} \frac{\lambda_{k}\left(\eta_{k}-t_{k}\right)^{\beta_{k}+1}}{\Gamma\left(\beta_{k}+2\right)}
$$$$
\times\left[\int_{t_{i-1}}^{t_{i}} \frac{\left(t_{i}-s\right)^{\alpha_{i-1}-2}}{\Gamma\left(\alpha_{i-1}-1\right)}\right.
$$$$
\times\left[a(s)+\xi_{1}|u(s)|^{\rho}+\xi_{2}|u(\theta(s))|^{\varrho}\right] d s
$$$$
\left.\left.+\left|I_{i}^{*}\left(u\left(t_{i}\right)\right)\right|\right]\right\}
$$$$
\leq\left[\|a\|+\xi_{1}\|u\|^{\rho}+\xi_{2}\|u\|^{\varrho}\right] \frac{\left(t-t_{k}\right)^{\alpha_{k}}}{\Gamma\left(\alpha_{k}+1\right)}
$$$$
+\sum_{i=1}^{k}\left[\left[\|a\|+\xi_{1}\|u\|^{\rho}+\xi_{2}\|u\|^{\varrho}\right] \frac{\left(t_{i}-t_{i-1}\right)^{\alpha_{i-1}}}{\Gamma\left(\alpha_{i-1}+1\right)}+L_{2}\right]
$$$$
+\sum_{i=1}^{k-1}\left(t_{k}-t_{i}\right)\left[\left[\|a\|+\xi_{1}\|u\|^{\rho}+\xi_{2}\|u\|^{\rho}\right]\right.
$$

$$
\left.\times \frac{\left(t_{i}-t_{i-1}\right)^{\alpha_{i-1}-1}}{\Gamma\left(\alpha_{i-1}\right)}+L_{3}\right]
$$




$$
\begin{aligned}
& +\sum_{i=1}^{k}\left(t-t_{k}\right)\left[\left[\|a\|+\xi_{1}\|u\|^{\rho}+\xi_{2}\|u\|^{\rho}\right]\right. \\
& \left.\times \frac{\left(t_{i}-t_{i-1}\right)^{\alpha_{i-1}-1}}{\Gamma\left(\alpha_{i-1}\right)}+L_{3}\right] \\
& +\Delta\left\{\left[\|a\|+\xi_{1}\|u\|^{\rho}+\xi_{2}\|u\|^{\rho}\right] \sum_{k=0}^{p} \frac{\lambda_{k}\left(\eta_{k}-t_{k}\right)^{\alpha_{k}+\beta_{k}}}{\Gamma\left(\alpha_{k}+\beta_{k}+1\right)}\right. \\
& +\sum_{k=1}^{p} \sum_{i=1}^{k} \frac{\lambda_{k}\left(\eta_{k}-t_{k}\right)^{\beta_{k}}}{\Gamma\left(\beta_{k}+1\right)} \\
& \times\left[\left[\|a\|+\xi_{1}\|u\|^{\rho}+\xi_{2}\|u\|^{\rho}\right]\right. \\
& \left.\times \frac{\left(t_{i}-t_{i-1}\right)^{\alpha_{i-1}}}{\Gamma\left(\alpha_{i-1}+1\right)}+L_{2}\right] \\
& +\sum_{k=1}^{p} \sum_{i=1}^{k-1} \frac{\lambda_{k}\left(\eta_{k}-t_{k}\right)^{\beta_{k}}\left(t_{k}-t_{i}\right)}{\Gamma\left(\beta_{k}+1\right)} \\
& \times\left[\left[\|a\|+\xi_{1}\|u\|^{\rho}+\xi_{2}\|u\|^{\varrho}\right]\right. \\
& \left.\times \frac{\left(t_{i}-t_{i-1}\right)^{\alpha_{i-1}-1}}{\Gamma\left(\alpha_{i-1}\right)}+L_{3}\right] \\
& +\sum_{k=1}^{p} \sum_{i=1}^{k} \frac{\lambda_{k}\left(\eta_{k}-t_{k}\right)^{\beta_{k}+1}}{\Gamma\left(\beta_{k}+2\right)} \\
& \times\left[\left[\|a\|+\xi_{1}\|u\|^{\rho}+\xi_{2}\|u\|^{\varrho}\right]\right. \\
& \left.\left.\times \frac{\left(t_{i}-t_{i-1}\right)^{\alpha_{i-1}-1}}{\Gamma\left(\alpha_{i-1}\right)}+L_{3}\right]\right\} \\
& \leq \Upsilon\|a\|+\left(1+\Lambda_{2}\right) p L_{2}+\left[(p-1) T \Lambda_{2}+p T \Lambda_{3}\right] L_{3} \\
& +\Upsilon \xi_{1}\|u\|^{\rho}+\Upsilon \xi_{2}\|u\|^{\varrho} \\
& \leq \mu(a)+\Upsilon \xi_{1}\|u\|^{\rho}+\Upsilon \xi_{2}\|u\|^{\varrho} .
\end{aligned}
$$

Thus,

$$
\|\mathscr{G} u\| \leq \mu(a)+\Upsilon \xi_{1}\|u\|^{\rho}+\Upsilon \xi_{2}\|u\|^{\varrho} \leq \frac{R}{3}+\frac{R}{3}+\frac{R}{3}=R .
$$

This implies $\mathscr{G}: \mathscr{B} \rightarrow \mathscr{B}$. Hence, we conclude that $\mathscr{G}:$ $\mathscr{B} \rightarrow \mathscr{B}$ is completely continuous. It follows from the Schauder fixed point theorem that the operator $\mathscr{G}$ has at least one fixed point. That is, problem (1) has at least one solution in $\mathscr{B}$.
Theorem 5. Suppose that there exist a nonnegative function $M \in C\left(J, \mathbb{R}^{+}\right)$and nonnegative constants $N, K$ such that

$$
\begin{gathered}
|f(t, u)-f(t, v)| \leq M(t)|u-v|, \\
\left|I_{k}(u)-I_{k}(v)\right| \leq N|u-v|, \\
\left|I_{k}^{*}(u)-I_{k}^{*}(v)\right| \leq K|u-v|,
\end{gathered}
$$

for $t \in J, u, v \in \mathbb{R}$ and $k=1,2, \ldots, p$. Furthermore, the assumption $\mu(M)<1$ holds. Then problem (1) has a unique solution.

Proof. For $u, v \in P C(J, \mathbb{R})$, we have

$$
\begin{aligned}
& |(\mathscr{G} u)(t)-(\mathscr{G} v)(t)| \\
& \leq \int_{t_{k}}^{t} \frac{(t-s)^{\alpha_{k}-1}}{\Gamma\left(\alpha_{k}\right)} \\
& \quad \times|f(s, u(s), u(\theta(s)))-f(s, v(s), v(\theta(s)))| d s \\
& +\sum_{i=1}^{k}\left[\int_{t_{i-1}}^{t_{i}} \frac{\left(t_{i}-s\right)^{\alpha_{i-1}-1}}{\Gamma\left(\alpha_{i-1}\right)}\right. \\
& \quad \times|f(s, u(s), u(\theta(s)))-f(s, v(s), v(\theta(s)))| d s \\
& \left.\quad+\left|I_{i}\left(u\left(t_{i}\right)\right)-I_{i}\left(v\left(t_{i}\right)\right)\right|\right]
\end{aligned}
$$$$
+\sum_{i=1}^{k-1}\left(t_{k}-t_{i}\right)\left[\int_{t_{i-1}}^{t_{i}} \frac{\left(t_{i}-s\right)^{\alpha_{i-1}-2}}{\Gamma\left(\alpha_{i-1}-1\right)}\right.
$$$$
\times \mid f(s, u(s), u(\theta(s)))
$$$$
-f(s, v(s), v(\theta(s))) \mid d s
$$$$
\left.+\left|I_{i}^{*}\left(u\left(t_{i}\right)\right)-I_{i}^{*}\left(v\left(t_{i}\right)\right)\right|\right]
$$$$
+\sum_{i=1}^{k}\left(t-t_{k}\right)\left[\int_{t_{i-1}}^{t_{i}} \frac{\left(t_{i}-s\right)^{\alpha_{i-1}-2}}{\Gamma\left(\alpha_{i-1}-1\right)}\right.
$$$$
\times \mid f(s, u(s), u(\theta(s)))
$$$$
-f(s, v(s), v(\theta(s))) \mid d s
$$$$
\left.+\left|I_{i}^{*}\left(u\left(t_{i}\right)\right)-I_{i}^{*}\left(v\left(t_{i}\right)\right)\right|\right]
$$

$$
\begin{aligned}
+\Delta\left\{\sum_{k=0}^{p} \lambda_{k} \int_{t_{k}}^{\eta_{k}}\right. & \frac{\left(\eta_{k}-s\right)^{\alpha_{k}+\beta_{k}-1}}{\Gamma\left(\alpha_{k}+\beta_{k}\right)} \\
\times & \mid f(s, u(s), u(\theta(s))) \\
& -f(s, v(s), v(\theta(s))) \mid d s
\end{aligned}
$$




$$
\begin{aligned}
& +\sum_{k=1}^{p} \sum_{i=1}^{k} \frac{\lambda_{k}\left(\eta_{k}-t_{k}\right)^{\beta_{k}}}{\Gamma\left(\beta_{k}+1\right)} \\
& \times\left[\int_{t_{i-1}}^{t_{i}} \frac{\left(t_{i}-s\right)^{\alpha_{i-1}-1}}{\Gamma\left(\alpha_{i-1}\right)} \mid f(s, u(s), u(\theta(s)))\right. \\
& -f(s, v(s), v(\theta(s))) \mid d s \\
& \left.+\left|I_{i}\left(u\left(t_{i}\right)\right)-I_{i}\left(v\left(t_{i}\right)\right)\right|\right] \\
& +\sum_{k=1}^{p} \sum_{i=1}^{k-1} \frac{\lambda_{k}\left(\eta_{k}-t_{k}\right)^{\beta_{k}}\left(t_{k}-t_{i}\right)}{\Gamma\left(\beta_{k}+1\right)} \\
& \times\left[\int_{t_{i-1}}^{t_{i}} \frac{\left(t_{i}-s\right)^{\alpha_{i-1}-2}}{\Gamma\left(\alpha_{i-1}-1\right)} \mid f(s, u(s), u(\theta(s)))\right. \\
& -f(s, v(s), v(\theta(s))) \mid d s \\
& \left.+\left|I_{i}^{*}\left(u\left(t_{i}\right)\right)-I_{i}^{*}\left(v\left(t_{i}\right)\right)\right|\right] \\
& +\sum_{k=1}^{p} \sum_{i=1}^{k} \frac{\lambda_{k}\left(\eta_{k}-t_{k}\right)^{\beta_{k}+1}}{\Gamma\left(\beta_{k}+2\right)} \\
& \times\left[\int_{t_{i-1}}^{t_{i}} \frac{\left(t_{i}-s\right)^{\alpha_{i-1}-2}}{\Gamma\left(\alpha_{i-1}-1\right)} \mid f(s, u(s), u(\theta(s)))\right. \\
& -f(s, v(s), v(\theta(s))) \mid d s \\
& \left.\left.+\left|I_{i}^{*}\left(u\left(t_{i}\right)\right)-I_{i}^{*}\left(v\left(t_{i}\right)\right)\right|\right]\right\} \\
& \leq\left\{\Upsilon\|M\|+\left(1+\Lambda_{2}\right) p L_{2}+\left[(p-1) T \Lambda_{2}\right.\right. \\
& \left.\left.+p T \Lambda_{3}\right] L_{3}\right\}\|u-v\| \\
& =\mu(M)\|u-v\| \text {. }
\end{aligned}
$$

As $\mu(M)<1$, we have $\|\mathscr{G} u-\mathscr{G} v\|<\|u-v\|$. Therefore, $\mathscr{G}$ is a contraction. It follows from the Banach contraction mapping principle that problem (1) has a unique solution.

Example 6. For $\alpha_{0}=5 / 4, \alpha_{1}=8 / 5, \beta_{0}=1 / 2, \beta_{1}=5 / 3$, $\lambda_{0}=2 / 5, \lambda_{1}=3 / 7, \eta_{0}=1 / 2, \eta_{1}=4 / 5,0<\rho, \varrho<1$, and $t_{1}=3 / 4$, we consider the following impulsive multi-orders fractional differential equation:

$$
\begin{aligned}
&{ }^{C} D_{t_{k}^{+}}^{\alpha_{k}} u(t)= \frac{e^{t} \sin ^{2}\left[3 u(t)+e^{(1 / 2) u(t)}\right]}{2+u^{4}(t)} \\
&+\frac{\cos (2 t+5)}{\sqrt{3+u^{2}(t)}}|u(t)|^{\rho}+\frac{\arctan ^{2} u(t)}{3}\left|u\left(t^{3}\right)\right|^{\varrho}, \\
& \quad 0<t<1, \quad t \neq \frac{3}{4}, k=0,1,
\end{aligned}
$$

$$
\begin{gathered}
\Delta u\left(\frac{3}{4}\right)=11 \sin ^{2} u\left(\frac{3}{4}\right), \quad \Delta u^{\prime}\left(\frac{3}{4}\right)=\frac{|u(3 / 4)|}{2(1+|u(3 / 4)|)}, \\
u(0)=\sum_{k=0}^{1} \lambda_{k} \mathcal{F}_{t_{k}^{+}}^{\beta_{k}} u\left(\eta_{k}\right)+\frac{1}{2}, \quad u^{\prime}(0)=0 .
\end{gathered}
$$

Observe that

$$
\begin{aligned}
|f(t, u, v)|= & \left.\left|\frac{e^{t} \sin ^{2}\left[3 u+e^{(1 / 2) u}\right]}{2+u^{4}}+\frac{\cos (2 t+5)}{\sqrt{3+u^{2}}}\right| u\right|^{\rho} \\
& +\frac{\arctan ^{2} u}{3}|v|^{\varrho} \mid \\
\leq & \frac{e^{t}}{2}+\frac{1}{\sqrt{3}}|u|^{\rho}+\frac{\pi}{12}|v|^{\varrho} .
\end{aligned}
$$

Clearly, $a(t)=e^{t} / 2, \xi_{1}=1 / \sqrt{3}, \xi_{2}=\pi / 12, L_{2}=11$, and $L_{3}=1 / 2$ and the conditions of Theorem 4 hold. Thus, by Theorem 4 , the impulsive multi-orders fractional boundary value problem (30) has at least one solution.

\section{Conflict of Interests}

The authors declare that they have no conflict of interests.

\section{Authors' Contribution}

All authors have equal contributions.

\section{Acknowledgments}

This work is supported by the NNSF of China (no. 61373174) and the Natural Science Foundation for Young Scientists of Shanxi Province, China (no. 20120211002-3).

\section{References}

[1] A. A. Kilbas, H. M. Srivastava, and J. J. Trujillo, Theory and Applications of Fractional Differential Equations, vol. 204 of North-Holland Mathematics Studies, Elsevier Science, Amsterdam, The Netherlands, 2006.

[2] V. Lakshmikantham, S. Leela, and J. V. Devi, Theory of Fractional Dynamic Systems, Cambridge Scientific Publishers, Cambridge, UK, 2009.

[3] J. Sabatier, O. P. Agrawal, and J. A. T. Machado, Eds., Advances in Fractional Calculus: Theoretical Developments and Applications in Physics and Engineering, Springer, Dordrecht, The Netherlands, 2007.

[4] D. Baleanu, K. Diethelm, E. Scalas, and J. J. Trujillo, Fractional Calculus Models and Numerical Methods, vol. 3 of Series on Complexity, Nonlinearity and Chaos, World Scientific, Boston, Mass, USA, 2012.

[5] E. Dinç and D. Baleanu, "Fractional wavelet transform for the quantitative spectral resolution of the composite signals of the active compounds in a two-component mixture," Computers \& Mathematics with Applications, vol. 59, no. 5, pp. 1701-1708, 2010. 
[6] B. Ahmad and S. Sivasundaram, "Existence results for nonlinear impulsive hybrid boundary value problems involving fractional differential equations," Nonlinear Analysis: Hybrid Systems, vol. 3, no. 3, pp. 251-258, 2009.

[7] A. Debbouche and D. Baleanu, "Controllability of fractional evolution nonlocal impulsive quasilinear delay integrodifferential systems," Computers \& Mathematics with Applications, vol. 62, no. 3, pp. 1442-1450, 2011.

[8] Y. Tian and Z. Bai, "Existence results for the three-point impulsive boundary value problem involving fractional differential equations," Computers \& Mathematics with Applications, vol. 59, no. 8, pp. 2601-2609, 2010.

[9] G. M. Mophou, "Existence and uniqueness of mild solutions to impulsive fractional differential equations," Nonlinear Analysis: Theory, Methods \& Applications, vol. 72, no. 3-4, pp. 1604-1615, 2010.

[10] C. Bai, "Impulsive periodic boundary value problems for fractional differential equation involving Riemann-Liouville sequential fractional derivative," Journal of Mathematical Analysis and Applications, vol. 384, no. 2, pp. 211-231, 2011.

[11] L. Zhang, G. Wang, and G. Song, "Existence of solutions for nonlinear impulsive fractional differentia equations of order $\alpha \in(2,3]$ with nonlocal boundary conditions," Abstract and Applied Analysis, vol. 2012, Article ID 717235, 26 pages, 2012.

[12] L. Zhang and G. Wang, "Existence of solutions for nonlinear fractional differential equations with impulses and antiperiodic boundary conditions," Electronic Journal of Qualitative Theory of Differential Equations, no. 7, pp. 1-11, 2011.

[13] Z. Liu, L. Lu, and I. Szántó, "Existence of solutions for fractional impulsive differential equations with $p$-Laplacian operator," Acta Mathematica Hungarica, vol. 141, no. 3, pp. 203-219, 2013.

[14] X. Zhang, X. Huang, and Z. Liu, "The existence and uniqueness of mild solutions for impulsive fractional equations with nonlocal conditions and infinite delay," Nonlinear Analysis: Hybrid Systems, vol. 4, no. 4, pp. 775-781, 2010.

[15] K. Balachandran, S. Kiruthika, and J. J. Trujillo, "Existence results for fractional impulsive integrodifferential equations in Banach spaces," Communications in Nonlinear Science and Numerical Simulation, vol. 16, no. 4, pp. 1970-1977, 2011.

[16] R. P. Agarwal, M. Benchohra, and S. Hamani, "A survey on existence results for boundary value problems of nonlinear fractional differential equations and inclusions," Acta Applicandae Mathematicae, vol. 109, no. 3, pp. 973-1033, 2010.

[17] A. Bouzaroura and S. Mazouzi, "An alternative method for the study of impulsive differential equations of fractional orders in a Banach space," International Journal of Differential Equations, vol. 2013, Article ID 191060, 12 pages, 2013.

[18] G. Wang, B. Ahmad, and L. Zhang, "Impulsive anti-periodic boundary value problem for nonlinear differential equations of fractional order," Nonlinear Analysis: Theory, Methods \& Applications, vol. 74, no. 3, pp. 792-804, 2011.

[19] G. Wang, B. Ahmad, and L. Zhang, "On nonlocal integral boundary value problems for impulsive non-linear differential equations of fractional order," Fixed Point Theory, vol. 15, pp. 265-284, 2014.

[20] M. Fečkan, Y. Zhou, and J. R. Wang, "On the concept and existence of solution for impulsive fractional differential equations," Communications in Nonlinear Science and Numerical Simulation, vol. 17, no. 7, pp. 3050-3060, 2012.

[21] A. Chauhan and J. Dabas, "Local and global existence of mild solution to an impulsive fractional functional integrodifferential equation with nonlocal condition," Communications in Nonlinear Science and Numerical Simulation, vol. 19, no. 4, pp. 821-829, 2014.

[22] X.-F. Zhou, S. Liu, and W. Jiang, "Complete controllability of impulsive fractional linear time-invariant systems with delay," Abstract and Applied Analysis, vol. 2013, Article ID 374938, 7 pages, 2013.

[23] G. Wang, B. Ahmad, and L. Zhang, "Some existence results for impulsive nonlinear fractional differential equations with mixed boundary conditions," Computers \& Mathematics with Applications, vol. 62, no. 3, pp. 1389-1397, 2011.

[24] G. Wang, B. Ahmad, L. Zhang, and J. J. Nieto, "Comments on the concept of existence of solution for impulsive fractional differential equations," Communications in Nonlinear Science and Numerical Simulation, vol. 19, no. 3, pp. 401-403, 2014. 


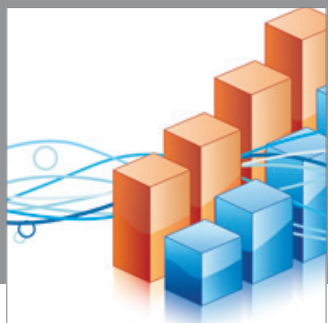

Advances in

Operations Research

mansans

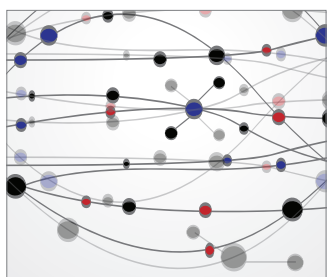

The Scientific World Journal
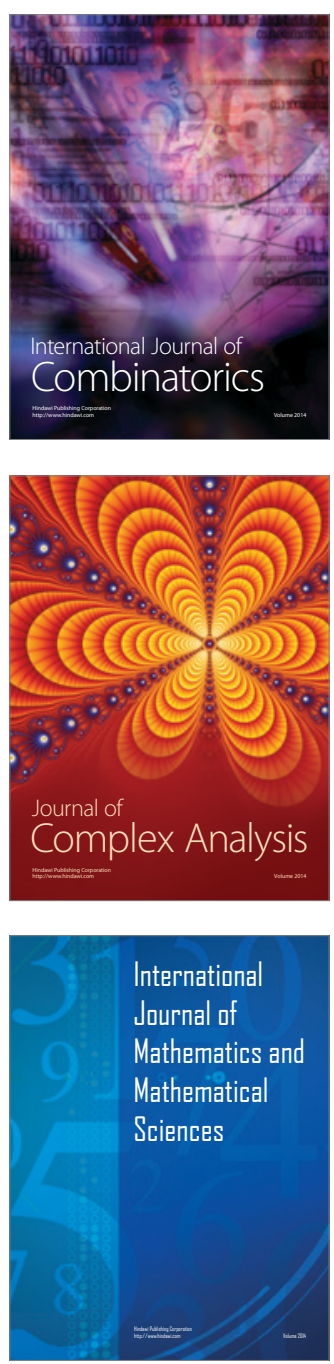
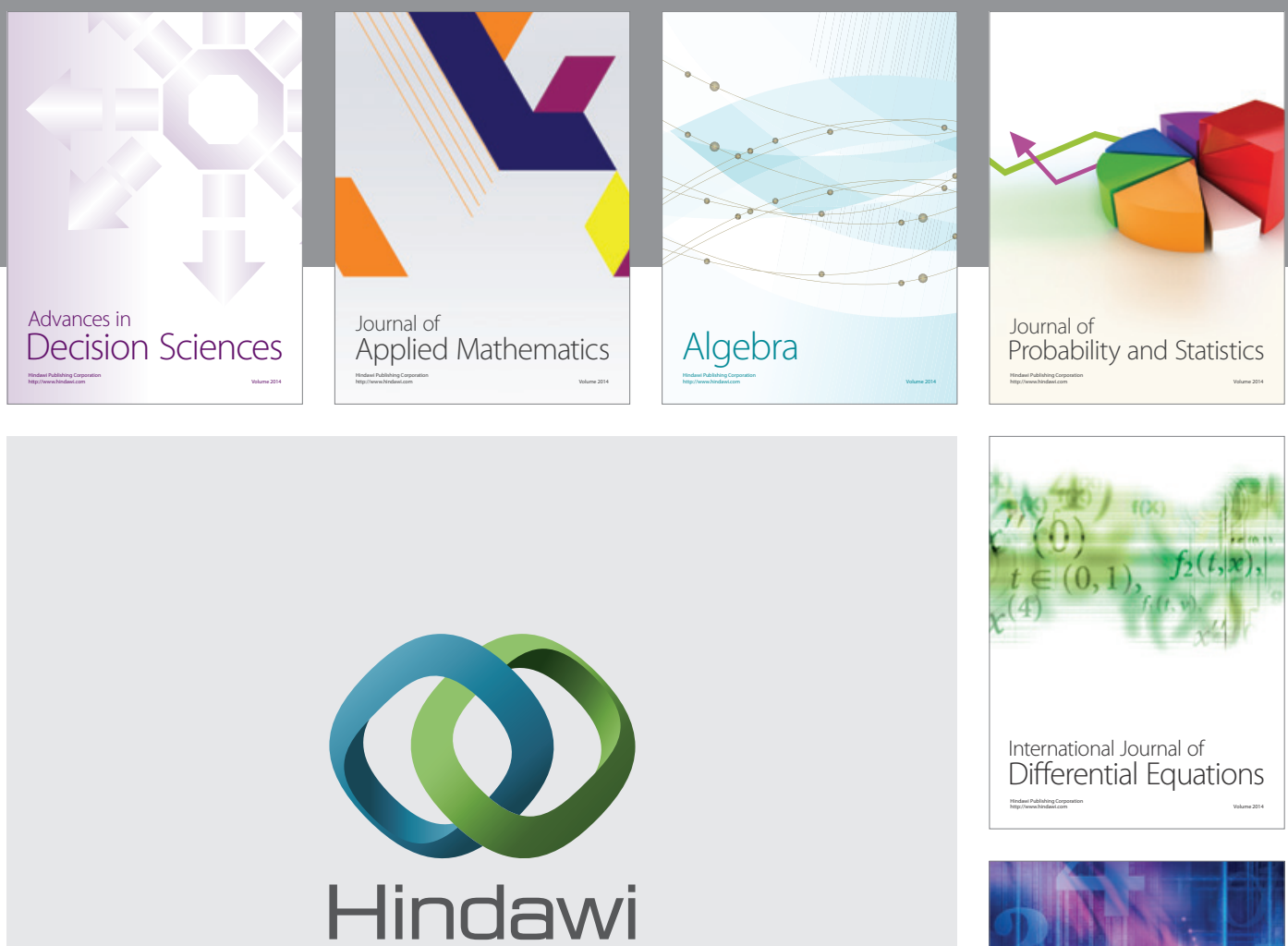

Submit your manuscripts at http://www.hindawi.com
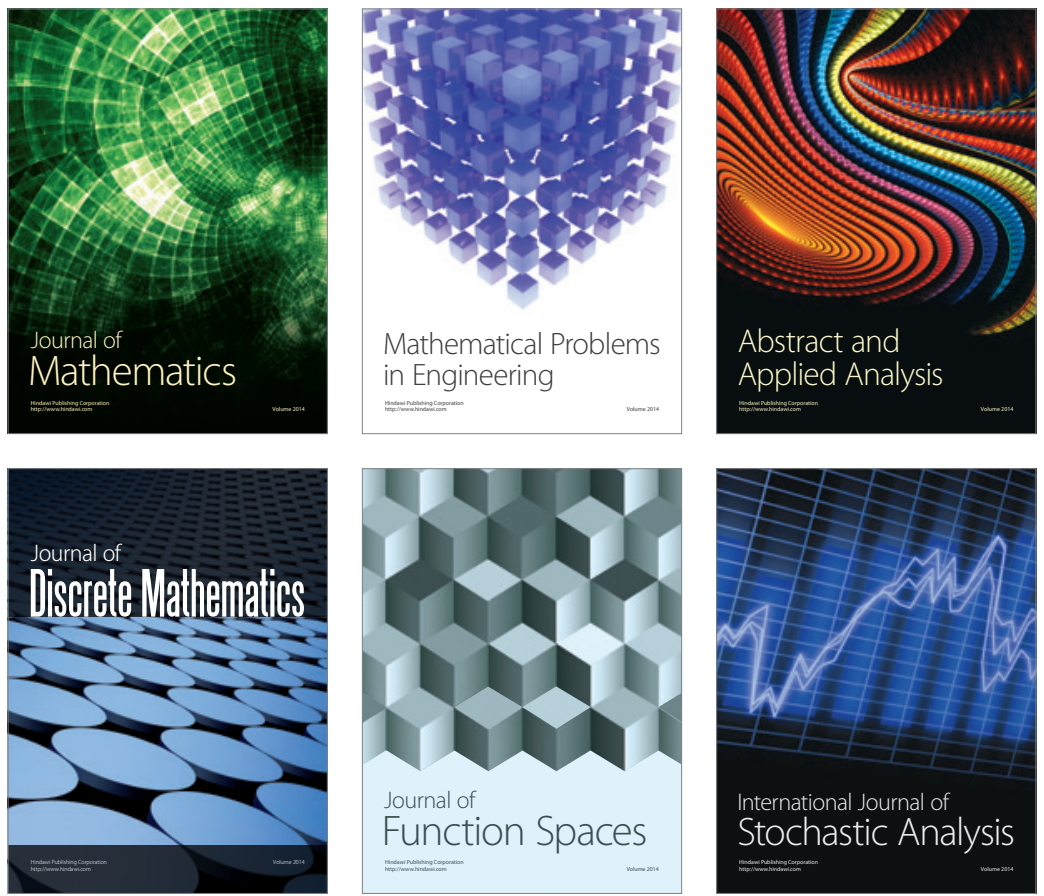

Journal of

Function Spaces

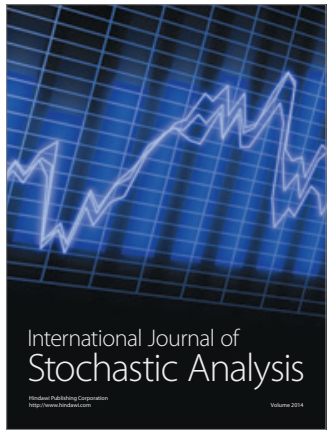

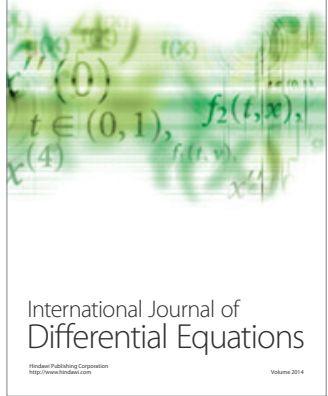
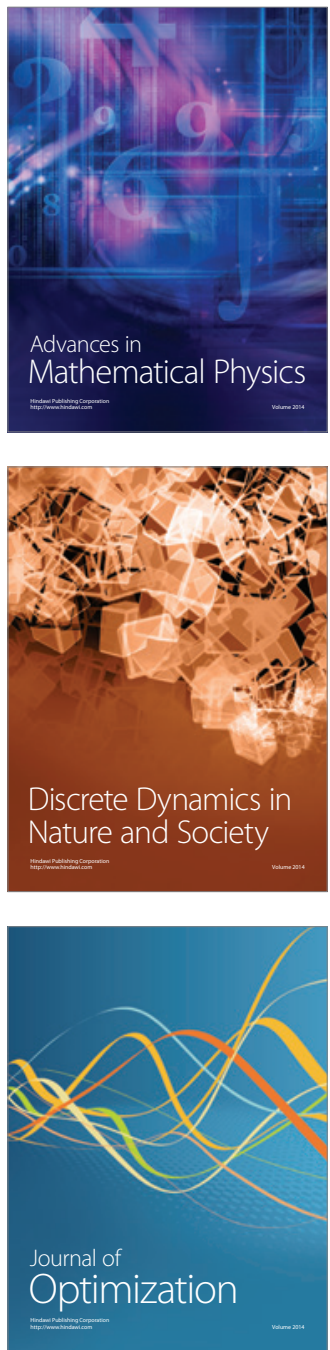